Rev Biomed 2003; 14:61-68.

\title{
Detección de VPH-16 y 18 en muestras de cérvix de mujeres que acuden a centros asistenciales de la ciudad de Mérida, Venezuela.
}

\section{Artículo Original}

Maritza Muñoz ${ }^{1}$, José A. Mendoza ${ }^{1}$, Luis Téllez ${ }^{1}$, María E. Noguera² $^{2}$ Orlando Moret ${ }^{3}$, Mercedes López $^{4}$, Morelva Toro ${ }^{4}$.

${ }^{1}$ Departamento de Microbiología y Parasitología Clínicas, ${ }^{2}$ Departamento de Ginecología y Obstetricia. Facultad de Medicina. ${ }^{3}$ Centro Clínico “Dr. Marcial Ríos". ${ }^{4}$ Cátedra de Citología, Facultad de Farmacia. Universidad de Los Andes. Mérida, Venezuela.

\section{RESUMEN.}

Introducción. El Virus del Papiloma Humano(VPH) produce infección del cuello uterino y se ha relacionado con cáncer del cérvix. El principal objetivo de este trabajo fue conocer la frecuencia de los subtipos VPH16 y 18 en mujeres venezolanas.

Material y métodos. Se estudiaron 271 mujeres que acudieron a diversos centros asistenciales de la ciudad de Mérida, Venezuela. Se obtuvieron muestras endo y exocervicales para la realización de la citología y la captura de híbridos $(\mathrm{CH})$ del ADN viral.

Resultados. 34 pacientes $(12,54 \%)$ resultaron positivas para VPH por $\mathrm{CH}$. El 88,24\% correspondió a VPH de alto riesgo; 18 casos fueron VPH-18, 9 fueron VPH-16, un caso presentó infección mixta y 2 no correspondieron a estos subtipos. De los casos positivos para VPH, 13 presentaron citología con alteraciones en células escamosas (Lesión intraepitelial escamosa (LIE) de bajo grado = 7; LIE de alto grado =6) y 8 pacientes atipias intracelulares indeterminadas. Los 13 casos restantes correspondieron a citologías normales y cambios celulares benignos. La citología demostró un valor predictivo negativo del $89 \%$.

Discusión. La frecuencia encontrada de infección por VPH es comparable con hallazgos de otros autores; sin embargo, existen diferencias en prevalencia debidas a las metodologías empleadas en cada estudio. Las diferencias estadísticas entre la detección viral y la Citología son contrarias a numerosas publicaciones, lo que significa subjetividad en la Citología. La elevada detección de VPH de alto riesgo, particularmente VPH-18 y 16, coincide con otros estudios, lo cual destaca la importancia de su detección temprana como parte de la prevención del cáncer uterino.

(Rev Biomed 2003; 14:61-68)

Palabras clave: Virus del Papiloma Humano, VPH16 y VPH-18, infección cervical, captura de híbridos

\section{SUMMARY.}

HPV-16 and 18 DNA detection from swabs of cervix of women who consulte at medical care centers in Merida City, Venezuela.

Introduction. Human papillomavirus (HPV) causes

Solicitud de sobretiros: Dra. Maritza Muñoz. Departamento de Microbiología y Parasitología Clínicas. Avenida 4 Bolívar con calle 32 Unda. Facultad de Medicina. 2do. Piso. Universidad de Los Andes. Mérida, 5101. Venezuela.

Tel. y FAX: 58-274-2403151. E-mail: mdelgado20@hotmail.com

Recibido el 8/Abril/2002. Aceptado para publicación el 30/Octubre/2002.

Este artículo está disponible en http://www.uady.mx/ biomedic/rb031422.pdf

Vol.14/No.2/Abril-Junio, 2003 


\section{Muñoz, JA Mendoza, L Téllez, ME Noguera, O Moret, M López, M Toro.}

infection of the cervix uteri and has been strongly related to cervical cancer. The principal objective of this work was to study the frequency of HPV-16 and HPV-18 in venezuelan women.

Material and methods. 271 female outpatients who attended several centers in Mérida-Venezuela, were studied. Endocervical and ectocervical specimens were obtained in order to perform the diagnostic procedures. Results. 34 patients (12.54\%) were HPV positive by $\mathrm{HC}, 30$ of whom corresponded to high risk HPV, 4 of low risk, and 4 were positive for both groups of risk. 18 patients had HPV-18, other 9 had HPV-16, 1 both types, and 2 other types. Citology detected scamous intraepithelial lesion (SIL) and indeterminate atypia intracellular in 21 patients with HPV. The remaining 13 cases had no cytological abnormalities. Citology showed $89 \%$ negative predictive value.

Discussion. The HPV infection frequency found in this work is comparable to other studies, nevertheless, there are several differences in prevalence due to different methodologies applied in each work. The significant differences between Citology and viral detection are contrary to the findings reported in numerous works. This can be explained by the subjectivity of citology. The high frequency of high risk HPV, particularly HPV-18 and 16, is similar to other works. This remarks the crucial role of HPV early detection as an important tool in the prevention of cancer of the cervix. (Rev Biomed 2003; 14:61-68)

Key words: HPV-16, HPV-18, infection of the cervix, hybrid capture system assay, cancer of the cervix.

\section{INTRODUCCIÓN.}

En Venezuela, el cáncer de cuello uterino es una de las primeras causas de muerte en mujeres, particularmente en edad reproductiva. Más de 45.000 muertes ocurrieron por cáncer de todos los tipos hasta el trienio 1990-1993, de los cuales el 17,2\% fueron por cáncer del cuello uterino (1). Este tipo de cáncer ocupa el primer lugar de morbilidad por tumores malignos en el estado Mérida, Venezuela, especialmente en mujeres de 45-64 años (2) y el tercer lugar de mortalidad por la misma causa y en el mismo grupo de edad (3).

La infección por el Virus del Papiloma Humano (VPH) ocasiona lesiones en el cuello uterino asociadas con el desarrollo de cáncer (4). Existen aproximadamente 20 subtipos de $\mathrm{VPH}$ relacionados con la infección cervical, de los cuales, catorce están más asociados con el desarrollo de cáncer y de éstos, los subtipos 16 y 18 son los más importantes (5-10). El genoma de estos subtipos está presente por lo general, en una configuración episomal intracelular, aunque puede integrarse al ADN de la célula hospedadora (7-10). Las proteínas virales E6 y E7, son responsables de la transformación celular, en virtud de la habilidad de unirse e inactivar las proteínas intracelulares p53 y Rb del hospedero. Estas proteínas de supresión tumoral son responsables de inhibir intracelularmente la acción de productos oncogénicos, detienen la progresión del ciclo celular y están involucradas en los procesos de apoptosis. Su función alterada por proteínas virales, genera fallas en la regulación de la fase $\mathrm{S}$ de la división celular y como consecuencia pueden desarrollarse eventos celulares que conllevan a malignidad $(7,10,11)$.

Para realizar el diagnóstico rutinario de infección cervical por VPH, se han empleado el examen ginecológico, que puede incluir colposcopía; el estudio citológico e histopatológico (12). Actualmente se utilizan métodos moleculares fundamentados en la detección del $\mathrm{ADN}$ viral, que además permiten tipificar VPH con elevada sensibilidad y especificidad (1318).

En vista de las fallas en los métodos tradicionales de diagnóstico y de las connotaciones clínicas y terapéuticas de la infección por VPH, se considera clave la inclusión de pruebas moleculares para detectar subtipos virales de alto riesgo de generar cáncer cervical, en particular VPH-16 y VPH-18, con la finalidad de adoptar medidas terapéuticas oportunas $(19,20)$.

Por todo lo anteriormente expuesto, se ha diseñado el presente estudio, cuyo principal objetivo es determinar la frecuencia de VPH de alto riesgo, particularmente los subtipos VPH-16 y 18, en

\section{Revista Biomédica}




\section{Detección de VPH-16 y 18 en mujeres venezolanas.}

muestras cervicales de mujeres que acudieron a las consultas de Ginecología de diversos centros asistenciales de la ciudad de Mérida, Venezuela, mediante el método de captura de híbridos $(\mathrm{CH})$ del ADN viral y comparar estos hallazgos con las características citológicas respectivas.

\section{MATERIAL Y MÉTODOS. Pacientes.}

Mediante un estudio clínico, observacional y de corte transversal, se evaluaron 271 mujeres mayores de 15 años de edad con actividad sexual, que acudieron a la clínica de prevención de cáncer de la Sociedad Anticancerosa, consulta de ginecología del Instituto Autónomo Hospital Universitario Los Andes y Centro Clínico “Dr. Marcial Ríos" en la ciudad de Mérida, Venezuela (junio 1996 - junio 2000). Se excluyeron pacientes con sangrado genital; procedimientos quirúrgicos previos que impidieran la adecuada toma de muestra; tratamiento inmunosupresor, seropositividad para VIH o embarazo diagnosticado. Luego de la realización de la historia clínica correspondiente, de acuerdo a un formato de recolección de datos ad hoc que incluyó entre otros, datos demográficos, historia familiar y personal, historia ginecológica, examen ginecológico (incluyendo colposcopía) y previo consentimiento de cada paciente, se llevó a cabo la toma de muestras endo y exocervical para su estudio.

\section{Toma y procesamiento de muestras.}

Se tomó muestra exocervical con espátula de Ayre y muestra endocervical con hisopo para la citología, realizada de acuerdo a la técnica de Papanicolaou $(12,21)$ y evaluada, de acuerdo a los criterios de la clasificación Bethesda (21), en la Cátedra de Citología de la Facultad de Farmacia de la Universidad de Los Andes. Luego, en el Laboratorio de Microbiología y Parasitología de la Facultad de Medicina de la misma Universidad, se tomó muestra endocervical, exocervical y de la zona de transformación con un hisopo de dacrón, el cual fue introducido en el medio de transporte ViraPap (Digene Diagnostics, Inc. Silver Spring, Maryland, USA) y almacenado en congelación a $-20^{\circ} \mathrm{C}$ hasta la realización del método de $\mathrm{CH}$ del $\mathrm{ADN}$ viral (Digene Diagnostics, Inc. Silver Spring, Maryland, USA).

El procesamiento de las muestras por $\mathrm{CH}$ se realizó según las instrucciones del fabricante y ha sido descrito anteriormente en otra publicación (22); brevemente: Una vez descongeladas las muestras se agregó el reactivo de desnaturalización y se incubó por 45 minutos a $65^{\circ} \mathrm{C}$; luego se procedió al paso de hibridación con una mezcla de sondas "A" de ARN correspondiente a subtipos de VPH de bajo riesgo y otra mezcla "B" (sondas de alto riesgo), durante una hora a $65^{\circ} \mathrm{C}$; se transfirieron las mezclas a tubos sensibilizados con anticuerpos monoclonales antihíbridos ADN/ARN para realizar el paso de "captura" por una hora y a temperatura ambiente; seguidamente se agregó un segundo anticuerpo monoclonal conjugado con fosfatasa alcalina y se incubó por 30 minutos a temperatura ambiente; finalmente y posterior a lavados, se añadió la mezcla de sustrato y luminol, para generar la señal de la reacción, la cual fue detectada por un quimioluminómetro (DCR-1, Digene Diagnostics, Inc.). El método incluye controles positivos para cada grupo de sondas y controles negativos con la finalidad de establecer el punto de corte y el control de calidad. Aquellas muestras que resultaron positivas para VPH de alto riesgo (VPH$16,18,31,33,35,45,51,52,56$ ), fueron nuevamente procesadas para detectar individualmente VPH-16 y VPH-18. Se eligió el método de $\mathrm{CH}$ para detectar estos subtipos virales, en virtud de que el procedimiento es similar al previamente descrito, los resultados son confiables y repetibles y además, la CH está aprobada por la FDA de Estados Unidos con fines diagnósticos. No obstante, debe señalarse que el método de $\mathrm{CH}$ no incluye todas las sondas para detectar los subtipos de VPH relacionados con la infección cervical y por ende, cabe la posibilidad de hallazgos falsos negativos.

\section{Análisis estadístico.}

Se realizó una base de datos en el programa EPI-Info versión $6.04 \mathrm{~b}$, convertida posteriormente a EPI-2000. Se empleó el análisis simple y estratificado en tablas de 2x2 incluidos en este programa (StatCalc y EpiTable). Para el análisis inferencial, se utilizó el estadístico $\mathrm{C}^{2}$, corregido por McNemar. 
M Muñoz, JA Mendoza, L Téllez, ME Noguera, O Moret, M López, M Toro.

\section{RESULTADOS.}

Se estudiaron 271 mujeres, con edad mínima de 16 años, máxima de 64 y un promedio de 30,7 años. El examen ginecológico en 260 pacientes (95,9\%), no mostró lesión alguna en genitales externos. Sólo 6 pacientes presentaron intértrigo y 4 presentaron condiloma acuminado en labios menores y un caso lo presentó en vagina.

En el cuadro 1 puede observarse que las muestras del cérvix procesadas por $\mathrm{CH}$ fueron positivas para VPH en 34 casos (12,54\%). La mayoría de las pacientes en quienes se detectó el ADN de VPH por este método, pertenecieron al grupo comprendido entre 15 y 35 años de edad (28 casos) y 6 al grupo de 36-64 años.

El 61,76\% de los casos positivos para VPH por $\mathrm{CH}$, fueron detectados en el grupo de pacientes cuya citologías mostraron alteraciones en células escamosas, es decir, lesión intraepitelial escamosa (LIE) y atipias celulares indeterminadas (ASCUS). El 38,24\% restante de los casos positivos para VPH, presentaron citologías sin anormalidad en células escamosas. La mayor proporción de casos positivos se detectó en pacientes con LIE de alto grado (LIE AG) (6 de 14 pacientes). Esta proporción disminuyó en la medida en que se encontraron menos alteraciones citológicas (cuadro 1).

Al analizar comparativamente la detección del ADN viral por $\mathrm{CH}$ y la Citología como método de tamizado, pudo demostrarse que la sensibilidad de la segunda para establecer el diagnóstico de VPH fue del 58,3\% (95\% IC: 41,5-72,6); especificidad del
60,4\% (95\% IC: $53,6-70,4)$ y un valor predictivo negativo del 89\% (95\% IC: 84,1-94,7). Se detectaron diferencias estadísticamente significativas entre la Citología y $\mathrm{CH}$ para el diagnóstico de $\mathrm{VPH}(\mathrm{p}<0,01)$; al tomar en cuenta aquellas citologías sospechosas de infección viral LIE de bajo grado (LIE BG).

De los 34 casos positivos para VPH por el método de $\mathrm{CH}$; 14 se detectaron en el grupo de edad de $<25$ años (41\%); 12 en el grupo de 25 - 34 años (35\%); 7 en el de 45 - 54 (21\%); y ninguno en el grupo de 55 - 64 años (figura 1).

En el cuadro 2 puede destacarse que 30 muestras $(88,24 \%)$ de las 34 positivas para VPH, incluyendo pacientes con infección mixta, correspondieron a VPH de alto riesgo para generar cáncer de cérvix; mientras que sólo 4 pacientes presentaron VPH de bajo riesgo exclusivamente.

Se realizó tipificación definitiva para VPH-16 y VPH-18 sólo en estas 30 pacientes positivas para VPH de alto riesgo y pudo evidenciarse que 18 casos (60\%) presentaron infección cervical por VPH-18; 9 casos $(30 \%)$ con VPH-16; un solo caso mostró infección mixta por VPH-16 y VPH-18 y dos casos (6,7\%), no correspondieron con estos subtipos virales (cuadro 3).

\section{DISCUSIÓN.}

La puesta en práctica de la $\mathrm{CH}$ en células exfoliadas de cuello uterino en 271 pacientes, mostró una frecuencia de infección por VPH del 12,54\% en mujeres que han iniciado vida sexual y con diferentes resultados citológicos en la muestra de población

Cuadro 1

Distribución de los casos de VPH en relación con los hallazgos citológicos.

\begin{tabular}{lccc}
\hline Diagnóstico citológico & $\mathrm{N}^{\mathrm{o}}$ de pacientes & CH positiva & $\%$ \\
\hline *L.I.E. alto grado & 14 & 6 & 42,86 \\
*L.I.E. bajo grado & 43 & 7 & 16,28 \\
Atipias indeterminadas & 52 & 8 & 15,38 \\
Cambios celulares benignos & 126 & 11 & 8,73 \\
Dentro de límites normales & 36 & 2 & 5,55 \\
\hline
\end{tabular}

*L.I.E.: Lesión intraepitelial escamosa

\section{Revista Biomédica}


Detección de VPH-16 y 18 en mujeres venezolanas.

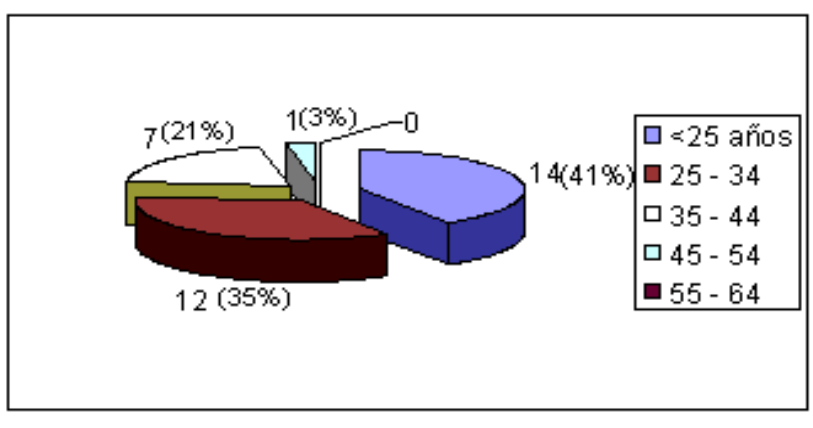

Figura 1.- Distribución de 34 casos positivos a VPH por $\mathrm{CH}$ de acuerdo a grupos de edad.

estudiada. En otras publicaciones puede observarse que la frecuencia de infección por VPH varía entre el $4 \%$ y el $70 \%(8,23-29)$, lo cual puede explicarse por el tipo de población seleccionada en estos estudios y por las condiciones socioculturales y sanitarias de cada región en particular. El porcentaje detectado en este trabajo puede explicarse además, porque incluyó mujeres que asistieron a consulta para control ginecológico periódico y se excluyeron aquellas pacientes con diagnóstico citológico o colposcópico de cáncer de cuello uterino. Sin embargo, existe una publicación de trabajo realizado en Venezuela en donde se reporta una mayor frecuencia de infección cervical por VPH (46,67\%), aun cuando se realizó en mujeres con cuello aparentemente sano (30).

El análisis sobre la detección del ADN viral por $\mathrm{CH}$ en relación a los diferentes grados de alteración citológica evidenció que la positividad a VPH aumenta en la medida en que se demuestra un mayor grado de lesión, en concordancia con lo publicado por otros

Cuadro 2

Distribución de 34 casos positivos para VPH de acuerdo a la clasificación en alto y bajo riesgo de producir cáncer de cuello uterino.

\begin{tabular}{|c|c|c|c|}
\hline $\begin{array}{l}\text { Captura de Híbridos } \\
\text { para VPH }\end{array}$ & $\begin{array}{c}\mathrm{N}^{\mathrm{o}} \text { de } \\
\text { pacientes }\end{array}$ & $\%$ & $\%$ acumulado \\
\hline Positivo (alto riesgo) & 26 & 76,48 & 76,48 \\
\hline Positivo (alto y bajo riesgo) & 4 & 11,76 & 88,24 \\
\hline Positivo (bajo riesgo) & 4 & 11,76 & 100 \\
\hline Total & 34 & 100 & \\
\hline
\end{tabular}

Cuadro 3

Frecuencia de los subtipos 16 y 18 en 30 pacientes positivas a VPH de alto riesgo.

\begin{tabular}{lcc}
\hline $\begin{array}{l}\text { Captura de Híbridos } \\
\text { para VPH }\end{array}$ & $\mathrm{N}^{\mathrm{o}}$ de pacientes \\
\hline VPH-18 & 18 & 60 \\
VPH-16 & 9 & 30 \\
VPH-16 y 18 & 1 & 3,3 \\
Otros subtipos & 2 & 6,7 \\
\hline Total & 30 & 100 \\
\hline
\end{tabular}

autores, empleando similar metodología (31-34). A pesar de que en la clasificación de Bethesda se considera a LIE BG equivalente a infección por VPH, debe destacarse que la detección de este virus por $\mathrm{CH}$ permitió demostrar la presencia de su genoma en pacientes cuyo resultado citológico se clasificaba en otras categorías, resaltando una mayor proporción en aquellos casos con LIE AG. Igualmente es importante señalar la detección del virus en pacientes con diagnóstico citológico de cambios celulares benignos y dentro de límites normales. Estos hallazgos son comparables a resultados publicados por otros autores $(29,31,33,35)$. Por otro lado, la comparación entre el diagnóstico de VPH por CH y la Citología mostró diferencias estadísticamente significativas, especialmente en pacientes con alteraciones citológicas de bajo grado (de 43 pacientes con diagnóstico citológico de LIE BG, se detectó VPH por $\mathrm{CH}$ sólo en 7), a diferencia de lo reportado por otros autores quienes demuestran una elevada correlación entre la detección viral y la presencia de $\operatorname{LIE~BG~(8,~20,~34,~}$ 36-39). Estas diferencias pueden explicarse por la variabilidad existente entre laboratorios y observadores, así como la conocida subjetividad de la Citología.

Por otra parte, existen evidencias que señalan a otros patógenos de cuello uterino, tales como Chlamydia trachomatis y virus Herpes simplex, como responsables de alteraciones importantes en células cervicales y su relación con el desarrollo de cáncer (40-43). Además, el método de CH empleado no incluye la detección de todos los subtipos de VPH

Vol.14/No. 2/Abril-Junio, 2003 


\section{Muñoz, JA Mendoza, L Téllez, ME Noguera, O Moret, M López, M Toro .}

asociados con la infección cervical, dejando así abierta la posibilidad de resultados falsos negativos, como ya se mencionara anteriormente.

En el presente trabajo, se ha encontrado un elevado porcentaje de VPH de alto riesgo para generar cáncer de cérvix, lo cual coincide con lo hallado por otros autores $(10,44)$. Sin embargo, existen numerosos reportes que señalan al VPH-16 con mayor frecuencia de infección que el 18, aunque ambos en conjunto, son considerados como los principales responsables de infección cervical asociada con alteración en células epiteliales escamosas y con mayor potencial carcinogénico que el resto de subtipos virales $(10,11,25,36)$.

Por otro lado, es importante resaltar que la gran mayoría de las pacientes infectadas por VPH (76\%) se hallaban en menores de 35 años de edad y en virtud de encontrarse infectadas por subtipos de alto riesgo, debe alertar en relación con la adopción de medidas de control y seguimiento oportunas y apropiadas. Estos resultados destacan la importancia de la utilización de métodos sensibles y específicos para la detección de VPH de alto riesgo, en particular los subtipos 16 y 18 , conocidos por su capacidad oncogénica.

Las diferencias encontradas entre el presente trabajo y otros estudios pueden explicarse porque son realizados en poblaciones con características particulares y además, debe aumentarse el tamaño de la muestra para realmente inferir que el VPH-18 es más frecuente en la población venezolana.

Se concluye que la citología cervical es un método subjetivo y de baja sensibilidad para establecer el diagnóstico de infección por VPH, mientras que la detección viral por $\mathrm{CH}$, es un método sensible para evidenciar infección cervical subclínica y puede ser útil en la identificación de pacientes con tipos específicos de VPH y riesgo de desarrollar cáncer cervical. Por lo tanto, es imperante la determinación de subtipos virales con la finalidad de establecer mejores criterios de control y seguimiento en pacientes infectadas, sumado a los hallazgos clínicos, citológicos y colposcópicos. Finalmente, deben ponerse en práctica métodos accesibles de detección viral por biología molecular como $\mathrm{CH}$, para mejorar la sensibilidad y especificidad del diagnóstico de infección del cérvix por este virus, aun en mujeres con citología normal.

\section{AGRADECIMIENTOS.}

Este trabajo pudo realizarse gracias a financiamiento suministrado por el FONACIT, de acuerdo a subvención $\mathrm{N}^{\circ}$ S1-96001789 y al CDCHT-ULA, de acuerdo a subvención $\mathrm{N}^{\circ}$ M-583-96-07-C. También debemos agradecer a todo el personal de la Sociedad Anticancerosa de Mérida, Venezuela por su incondicional colaboración para la realización de este estudio.

\section{REFERENCIAS.}

1.- Ministerio de Salud y Desarrollo Social (República Bolivariana de Venezuela). Anuarios de Epidemiología y Estadística Vital. Tomo II. 1999.

2.- Corporación de Salud del Estado Mérida (República Bolivariana de Venezuela). Coordinación Regional de Oncología. Registro regional de tumores del IAHULA y Unidad de Larga Estancia. 2000.

3.- Corporación de Salud del Estado Mérida (República Bolivariana de Venezuela). Tarjetas de mortalidad general EPI13-B de epidemiología regional. 2000.

4.- Vikki M, Pukkala E, Niemen P, Hakama M. Gynaecological infections as risk determinants of subsequent cervical neoplasia. Acta Oncol 2000; 39: 71-5.

5.- Lörincz AT, Reid R, Jenson AB, Greenberg MD, Lancaster W, Kurman R. Human papillomavirus infection of the cervix: relative risk associations of 15 common anogenital types. Obstet Gynecol 1992; 79: 328-37.

6.- Kuhn L, Denny L, Pollack A, Lörincz AT, Richart RM, Wright TC. Human papillomavirus DNA testing for cervical cancer screening in low-resource settings. J Natl Cancer Inst 2000; 92: 818-25.

7.- Cannistra S, Niloff M. Cancer of the uterine cervix. New Eng J Med 1996; 334: 1030-8.

8.- Bavin PJ, Giles JA, Hudson E, Williams D, Crow J, Griffith $\mathrm{PD}$, et al. Comparison of cervical cytology and the polymerase chain reaction for HPV 16 to identify women with cervical disease in a general practice population. J Med Virol 1992; 37: 8-12.

9.- Coutlée F, Bobo L, Abbass H, Dalabetta G, Hook N, Shah

\section{Revista Biomédica}




\section{Detección de VPH-16 y 18 en mujeres venezolanas.}

$\mathrm{K}$, et al. Detection of HPV-16 in cell lines and cervical lavage specimen by polymerase chain reaction enzyme immunoassay assay. J Med Virol 1992; 37: 22-9.

10.- Lambropoulos AF, Agorastos T, Frangoulides E, Karahaliou R, Bontis J, Dozi-Vassiliades I. Detection of human papillomavirus using the polymerase chain reaction and typing for HPV-16 and 18 in the cervical smears of greek women. J Med Virol 1994; 43: 228-30.

11.- Gissmann L. Linking HPV to cancer. Clin Obstet Gynecol 1989; 32: 141-7.

12.- Schneider V. Microscopic diagnosis of HPV infection. Clin Obstet Gynecol 1989; 32: 148-56.

13.- Lörincz AT. Molecular methods for the detection of human papillomavirus infection. Obstet Gynecol Clin North Am 1996; 23: 707-30.

14.- Lörincz AT, Schiffman MH, Jaffurs WJ, Marlowe J, Quinn AP, Temple GF. Temporal associations of human papillomavirus infection with cervical cytology abnormalities. Am J Obstet Gynecol 1990; 162: 645-51.

15.- Koutsky LA, Holmes KK, Critchlow CW, Stevens CE, Paavonen PA, Beckman AM, et al. A cohort study of the risk of cervical intraepithelial neoplasia grade 2 or 3 in relation to papillomavirus infection. N Engl J Med 1992; 327: 1272-8.

16.- Cox JT, Lörincz AT, Schiffman MH, Sherman ME, Cullen A, Kurman RJ. Human papillomavirus testing by hybrid capture appears to be useful in triaging women with a cytologic diagnosis of atypical squamous cells of undetermined significance. Am J Obstet Gynecol 1995; 172: 946-54.

17.- Cuzick J, Szarewski A, Terry G, Ho L, Hanby A, Maddox $\mathrm{P}$, et al. Human papillomavirus testing in primary cervical screening. Lancet 1995; 345: 1533-6.

18.- Verdon ME. Issues in the management of human papillomavirus genital disease. Am Fam Phys 1997; 55:181322.

19.- Robertson AJ, Anderson JM, Beck JS, Burnett RA, Howatson SR, Lee FD, et al. Observer variability in hystopathological reporting of cervical biopsy specimens. J Clin Pathol 1989; 42: 231-8.

20.- Sherman ME, Schiffman MH, Lörincz AT, Manos MM, Scott DR, Kurman RJ, et al. Toward objective quality assurance in cervical cytopathology. Correlation of cytopathologic diagnoses with detection of high risk human papillomavirus types. Am J Clin Pathol 1994; 102: 182-7.

21.- The Bethesda system for reporting cervical/vaginal cytologic diagnoses. Acta Cytologica 1993; 37: 115-24.

22.- Mendoza JA, Muñoz M, Vielma S, Noguera ME, López M, Toro M. Infección cervical por el virus del papiloma humano: diagnóstico por citología y por captura de híbridos del ADN viral. Rev Obstet Ginecol Venez 2000; 60: 103-7.

23.- Mugica-van-Herckenrode C, Malcolm AD, Coleman DV. Prevalence of human papillomavirus (HPV) infection in Basque Country women using slot-blot hybridization: a survey of women at low risk of developing cervical cancer. Int J Cancer 1992; 51: 851-86.

24.- Koss LG. HPV testing as a screening tool for cervical cancer. JAMA 2000; 283: 2525-6.

25.- Muñoz N, Bosch FX. Cervical cancer and human papillomavirus. Epidemiological evidence and perspectives for prevention. Salud Pública Mex 1997; 39: 274-82.

26.- Muñoz N, Bosch FX, de-Sanjosé S, Viladiu P, Tormo J, Moreo $\mathrm{P}$ et al. El virus del papiloma humano en la etiología del cáncer cérvico-uterino. Bol Of Sanit Panam 1993; 115: 301-8.

27.- Kurz J, Mitra K, Adam R, Miao X, Mackay JS, Isa NN, et al. PCR detection and typing of genital papillomavirus in a New Brunswick population. Inter J Cancer 1993; 55: 604-8.

28.- Ramael M, Segers K, Pannemans N, Wesling F, vanMarck E. Detection of human papillomavirus in cervical scraping by in situ hibridization and the polymerase chain reaction in relation to citology. Histochem J 1995; 27: 57-9.

29.- Herrero R, Hildesheim A, Bratti C, Sherman ME, Hutchinson M, Morales J, et al. Population-based study of human papillomavirus infection and cervical neoplasia in rural Costa Rica. J Natl Cancer Inst 2000; 92: 464-74.

30.- Romero M, Ceballos M, Correnti M, Durán Z. Detección y tipificación del virus de papiloma humano en muestras de cuello uterino normal. Acta Cien Venez 1999; 50 (Supl-2): 205.

31.- Apgar BS, Brotzman G. HPV testing in the evaluation of the minimally abnormal Papanicolaou smear. Am Fam Phys 1999; 59: 2794-801.

32.- Ratnam S, Franco EL, Ferenczy A. Human papillomavirus testing for primary screening of cervical cancer precursors. 


\section{Muñoz, JA Mendoza, L Téllez, ME Noguera, O Moret, M López, M Toro.}

Cancer Epi Bio Prev 2000; 9: 945-51.

33.- Nuovo J. Detection of human papillomavirus in Papanicolaou smears: correlation with pathologic findings and clinical outcome. Diagn Mol Pathol 1998; 7: 158-63.

34.- Bergeron C, Jeannel D, Poveda J, Cassonnet P, Orth G. Human papillomavirus testing in women with mild cytologic atypia. Obstet Gynecol 2000; 95: 821-7.

35.- Clavel C, Masure M, Bory J-P, Putaud I, Mangeonjean C, Lorenzato M, Nazeyrollas P, Gabriel R, Quereux C, Birembaut P. Human papillomavirus testing in primary screening for the detection of high-grade cervical lesions: a study of 7932 women. B J Cancer 2001; 89: 1616-23.

36.- Burk RD, Kadish AS, Calderin S, Rommey SL. Human papillomavirus infection of the cervix detected by cervicovaginal lavage and molecular hybridization: correlation with biopsy and papanicolaou smear. Am J Obstet Gynecol 1986; 154: 982-9.

37.- de-Sanjosé S, Santamaría M, Alonso-de-Ruiz P, Aristizábal N, Guerrero E, Castellsague X, et al. HPV types in women with normal cervical cytology. IARC Sci Publ 1992; 119: 75-84.

38.- Farthing A, Masterson P, Mason WP, Vousden KH. Human papillomavirus detection by hybrid capture and its possible clinical use. J Clin Pathol 1994; 47: 649-52.

39.- Schneider A, Zahm DM, Kirchmayr R, Schneider VL. Screening for cervical intraepithelial neoplasia grade $2 / 3$ : validity of cytologic study, cervicography and human papillomavirus detection. Am J Obstet Gynecol 1996; 174: 1534-41.

40.- González-Sánchez JL, Flores-Avilés Y, Gómez-Campos G, Montero-Ramírez A. Association of Chlamydia trachomatis and human papillomavirus as predisposing factors in cervical intraepithelial neoplasia. Ginecol Obstet Mex 1995; 63: 422-6.
41.- Syrjanen K, Mantyjarvi R, Vayrynen M, Castren O, Yliskoski M, Saarikoski S. Chlamydial cervicitis in women followed-up for human papillomavirus (HPV) lesions of the uterine cervix. Acta Obstet Gynecol Scand 1985; 64: 467-71.

42.- Allerding TJ, Jordan SW, Boardman RE. Association of human papillomavirus and Chlamydia infections with incidence cervical neoplasia. Acta Cytol 1985; 29: 653-60.

43.- Balbi C, Di-Grazia F, Piscitelli V, Martini S, Cardone A, Balbi GC. Retrospective study of cervical papillomavirus lesions: early Herpes simplex virus proteins as markers of risk for progression. Minerva Ginecol 1996; 48: 175-9.

44.- Hatch KD, Schneider A, Abdel-Nour M. An evaluation of human papillomavirus testing for intermediate and highrisk types as triage before colposcopy. Am J Obstet Gynecol 1995; 172: 1150-7.

\section{Revista Biomédica}

